\title{
Fatal child maltreatment associated with multiple births in Japan: nationwide data between July 2003 and March 2011
}

\author{
Syuichi Ooki
}

Received: 13 February 2013/Accepted: 7 March 2013/Published online: 5 April 2013

(C) The Japanese Society for Hygiene 2013

\begin{abstract}
Objectives The purpose of the present study is to clarify the impact of multiple births in fatal child maltreatment (child death due to maltreatment).

Methods The national annual reports on fatal child maltreatment, which contain all cases from July 2003 to March 2011, published by the Ministry of Health, Labor and Welfare of Japan, were used as the initial sources of information. Parent-child murder-suicide cases were excluded from the analyses. Multiple births, teenage pregnancy and low-birthweight were regarded as the exposed groups. The relative risks (RRs) and their $95 \%$ confidence intervals (CIs) were estimated using the data from the above reports and vital statistics. These analyses were performed both including and excluding missing values.

Results Among 437 fatal child maltreatment cases, 14 multiple births from 13 families were identified. The RRs of multiple births per individual were 1.8 (95\% CI 1.0-3.0) when including missing values and 2.7 (95\% CI 1.5-4.8) when excluding missing values. The RRs of multiple births per family were 3.6 (95\% CI 2.1-6.2) when including missing values and 4.9 (95\% CI 2.7-9.0) when excluding missing values. The RR tended to be much lower than the RR of teenage pregnancy (RR 12.9 or 22.2), but slightly higher than the RR of low-birthweight (RR 1.4 or 2.9).

Conclusions Families with multiple births had elevated risk for fatal child maltreatment both per individual and per family unit. Health providers should be aware that multiple
\end{abstract}

\section{S. Ooki (ه)}

Department of Health Science,

Ishikawa Prefectural Nursing University,

1-1 Gakuendai, Kahoku, Ishikawa 929-1210, Japan

e-mail: sooki@ishikawa-nu.ac.jp pregnancies/births may place significant stress on families and should provide appropriate support and intervention.

Keywords Fatal child maltreatment - Families with multiple births - Teenage pregnancy - Low-birthweight . Nationwide data

\section{Introduction}

Multiple births are thought to be a risk factor for child maltreatment [1-4]. These earlier studies, however, were performed two to three decades ago, and were not necessarily population-based. It is of little doubt that the current conditions surrounding families, for example, family planning, child rearing practices and maternal/paternal age, are quite different from those prevalent at that time. Recently, family size has rapidly become smaller, maternal and paternal ages at first childbirth are becoming higher, and assisted reproductive technology has spread widely in Japan [5].

Nevertheless, very few population-based data on the relationship between child maltreatment and multiple births are available. In an intensive literature search, the present author could find no report on this topic other than the earlier studies mentioned above. One possible reason is that prospective epidemiologic research on child maltreatment is very difficult due to the underreporting of abuse cases. It has long been believed in Japan that the frequency of child maltreatment in cases of multiple births is around 10-fold higher than among singletons according to the only hospital-based report done in Japan, authored by Tanimura et al. [4]. The purpose of the present study is to clarify the impact of multiple births on fatal child maltreatment using nationwide data. 


\section{Materials and methods}

\section{Subjects}

National annual reports on fatal child maltreatment (the first to eighth reports) published by the Ministry of Health, Labor and Welfare of Japan (in Japanese) were used as the initial sources of information for the present secondary data analyses. All cases of fatal maltreatment of children from 0 to 17 years of age between July 2003 and March 2011 were reported.

Fatal child maltreatment was defined as child death due to maltreatment. The definitions of maltreatment and parental guardian were based on the Child Abuse Prevention Law of Japan executed in 2000. The types of maltreatment included physical abuse, psychological abuse, neglect and sexual abuse. The annual report tallied the cases of fatal child maltreatment according to whether the deaths were based on parent-child murder-suicide or not. Cases of parent-child murder-suicide were excluded from the present analysis, since the background and potential risk factors may be quite different from those in cases of fatal child maltreatment without suicide.

The numbers of women exhibiting any of about 20 physical and mental issues during pregnancy and the perinatal period were surveyed via questionnaire for the local public authorities and the results were presented in the annual reports. The reported number of women with each issue do not necessarily show that that particular issue is a real risk factor for fatal child maltreatment, since the frequency of each issue in the unexposed population or general population was not taken into consideration in the report. These data on physical and mental issues were not presented according to the ages of the victims. One limitation of this retrospective questionnaire survey is that there were many missing values among these data.

\section{Statistical analyses}

Multiple births, low-birthweight $(<2,500 \mathrm{~g})$ and teenage pregnancy were the only variables for potential risk factors, the numbers of which in the general population at birth could be estimated using vital statistics. The author substituted childbirth below the maternal age of 20 in the vital statistics for teenage pregnancy.

The relative risks (RRs) and their $95 \%$ confidence intervals (CIs) in cases of fatal child maltreatment related to multiple births were estimated using fatal maltreatment data and vital statistics. The RRs of teenage pregnancy and low-birthweight were also calculated to clarify the relative impact of multiple births on fatal child maltreatment. The data on multiple births and low-birthweight were presented in all eight reports, and teenage pregnancy was tracked beginning with the third annual report. The information on the missing values of teenage pregnancy, low-birthweight and multiple births were presented after the second report.

The RR was calculated as the ratio of the incidence in the exposed population to that in the unexposed population according to the definition. Multiple births, teenage pregnancy and low-birthweight were regarded as risk factors against singleton births, non-teenage pregnancy and nonlow-birthweight, respectively. The analyses were performed using the concept of the birth-year cohort. For example, the incidence in multiple births was calculated as the number of multiple births cases with fatal child maltreatment divided by the person-years of the birth-year cohort of the general multiple births population in the reported period (between July 2003 and March 2011). The incidence in singletons was calculated in the same manner. There were no data on the number of multiple births, birthweight or maternal age for children from one to 17 years of age in the vital statistics. It was assumed that the percentage of the exposed population in the total general population at birth was constant for children from one to 17 years of age. For example, the percentage of multiple births in 2003 was used as the percentage of multiples of 1 year of age in 2004, 2 years of age in 2005, and so on. For the general population data, vital statistics from 1986 to 2011 were used, considering the year of the annual report and the age of the victims. Theoretically, the victims of 17 years of age in the first report (published in 2005) were born in 1986, and the victims of 0 years of age in the eighth report (published in 2012) were born in 2011. The follow-up period of the birth-year cohort was adjusted for the years 2003 and 2011 according to the research period (6 months and 3 months, respectively). The follow-up period of multiple births was distributed from 0.125 years (2011 cohort) to 6.25 years (1994-2004 cohorts) according to the birth year. Then the RR was calculated as the ratio of the incidence in the multiple births population to that in the singleton population. The RRs of low-birthweight and teenage pregnancy were calculated in the same manner.

Regarding multiple births, all families with at least two live multiple births were recalculated using vital statistics of live births/stillbirths combination. The RR and $95 \%$ CI of multiple births were calculated per child unit (multiples as an individual child) and per family unit (families with multiples). When calculating RR per family, the total number of families was adjusted by considering the numbers of families with multiples.

These analyses were performed both including and excluding missing values, since a very high number of missing values was expected. Missing values were treated as unexposed cases when missing values were included. 


\section{Results}

The total number of cases of fatal child maltreatment in the reported period was 437 . The total number of person-years for children aged 0-17 years between July 2003 and March 2011 was estimated to be $159,550,946$ in Japan. The estimated mortality rate due to maltreatment of children aged 0-17 years was 0.27 per 100,000 person-years.

The percentages of missing values for multiple births, low-birthweight and teenage pregnancy were $39.1 \%$ $(=161 / 412), 50.5 \%(=208 / 412)$ and $35.1 \%(=127 / 362)$, respectively. Among cases of fatal child maltreatment, 14 multiple births were identified from 13 families.

The RRs and their $95 \%$ CIs are shown in Table 1. All RRs were statistically significant regardless of the risk factors and estimation methods, and were strongly influenced by the inclusion/exclusion of missing values. The RRs of multiple births per individual were $1.8(95 \%$ CI 1.0-3.0) when including missing values and 2.7 (95\% CI 1.5-4.8) when excluding missing values. The RRs of multiple births per family were 3.6 (95 \% CI 2.1-6.2) when including missing values and 4.9 (95 \% CI 2.7-9.0) when excluding missing values. The RR tended to be much lower than the RR of teenage pregnancy, (RR 12.9, $95 \% \mathrm{CI}$ 9.7-17.0 when including missing values, RR 22.2, $95 \% \mathrm{CI}$ 16.6-29.8 when excluding missing values) but slightly higher than the RR of low-birthweight (RR 1.4, $95 \% \mathrm{CI}$ 1.1-1.9 when including missing values, RR 2.9, $95 \% \mathrm{CI}$ 2.0-4.0 when excluding missing values).

\section{Discussion}

According to the world report by UNICEF [6], the maltreatment death rates of children under the age of 15 ranged from the lowest rates of $0.1-0.2$ to the highest rate of more than 2.0 (per 100,000 person-years) in the richest 27 countries in the 1990s. The estimated mortality rate due to maltreatment of children under the age of 15 years was $0.32(=422 / 130,716,055)$ per 100,000 person-years in the present study. It should be noted that this mortality rate does not include parent-child murder-suicide cases. If murder-suicide cases were included, the mortality rate is nearly $0.55(=723 / 130,716,055)$, thus demonstrating that there is no serious underreporting of fatal child maltreatment in the present data.

On the other hand, the incidence rate of total, including nonfatal, child maltreatment was difficult to estimate. One possible estimate can be made as follows. According to the report by the Ministry of Health, Labor and Welfare of Japan (in Japanese), the number of individuals using the listening and support services for child maltreatment in 206

Table 1 Relative risks and their $95 \%$ confidence intervals for teenage pregnancy, low-birthweight and multiple births

\begin{tabular}{|c|c|c|c|c|c|c|c|c|}
\hline & $\begin{array}{l}\text { Fatal child } \\
\text { maltreatment } \\
\text { (including } \\
\text { missing values }{ }^{\mathrm{a}} \text { ) } \\
N\end{array}$ & $\begin{array}{l}\text { Vital statistics } \\
\text { Person-year }\end{array}$ & $\mathrm{RR}$ & $95 \% \mathrm{CI}$ & $\begin{array}{l}\text { Fatal child } \\
\text { maltreatment } \\
\text { (excluding } \\
\text { missing values) } \\
N\end{array}$ & $\begin{array}{l}\text { Vital statistics } \\
\text { Person-year }\end{array}$ & $\mathrm{RR}$ & $95 \% \mathrm{CI}$ \\
\hline Age of pregnancy & & $(2005.1-2011.3)^{\mathrm{b}}$ & & & & $(2005.1-2011.3)^{\mathrm{b}}$ & & \\
\hline $20-$ & 302 & $125,753,388$ & 1 & Reference & 175 & $125,753,388$ & 1 & Reference \\
\hline-19 & 60 & $1,942,624$ & 12.9 & $9.7-17.0$ & 60 & $1,942,624$ & 22.2 & $16.6-29.8$ \\
\hline Birthweight & & $(2003.7-2011.3)^{\mathrm{b}}$ & & & & $(2004.1-2011.3)^{\mathrm{b}}$ & & \\
\hline Non-low-birthweight & 389 & $146,784,459$ & 1 & Reference & 163 & $136,849,333$ & 1 & Reference \\
\hline Low-birthweight & 48 & $12,766,487$ & 1.4 & $1.1-1.9$ & 41 & $11,994,708$ & 2.9 & $2.0-4.0$ \\
\hline Plurality & & $(2003.7-2011.3)^{\mathrm{b}}$ & & & & $(2004.1-2011.3)^{\mathrm{b}}$ & & \\
\hline \multicolumn{9}{|l|}{ Per individual child } \\
\hline Singleton & 423 & $156,642,370$ & 1 & Reference & 239 & $146,110,271$ & 1 & Reference \\
\hline Multiple births & 14 & $2,908,576$ & 1.8 & $1.0-3.0$ & 12 & $2,733,769$ & 2.7 & $1.5-4.8$ \\
\hline \multicolumn{9}{|l|}{ Per family } \\
\hline Families with singleton(s) & 390 & $156,642,370$ & 1 & Reference & 239 & $146,110,271$ & 1 & Reference \\
\hline Families with multiples & 13 & $1,454,839$ & 3.6 & $2.1-6.2$ & 11 & $1,367,332$ & 4.9 & $2.7-9.0$ \\
\hline
\end{tabular}

$R R$ relative risk, $C I$ confidence interval

${ }^{\text {a }}$ Missing values were treated as unexposed cases

${ }^{\mathrm{b}}$ Follow-up period is shown in parentheses 
child consultation centers throughout Japan was 59,919 in 2011. The incidence rate of child maltreatment was roughly estimated to be $295(=59,919 / 20,339,000)$ per 100,000 children under the age of 18 in the same year. If murder-suicide cases were included in the present calculation, the mortality rate of fatal child maltreatment under the age of 18 became nearly $0.47(=751 / 159,550,946)$ per 100,000 . Thus, cases of fatal child maltreatment were relatively rare among all cases of child maltreatment.

The present data showed that families with multiple births had an increased risk of fatal child maltreatment. The $\mathrm{RR}$, however, was not higher than the RR of teenage pregnancy. The results also showed that the RR of multiples per individual, namely of being a child member of multiple births, showed marginal significance and was not largely different from the RR of low-birthweight when missing values were included in the calculation.

The first reports that treated the relationship between families with multiples (twins in this case) and child maltreatment were that of Robarge et al. [1] and their expanded study [2]. However, their research interest was not necessarily twins as a risk factor for child maltreatment, but the stressful situation associated with the birth of twins due to the increase in family members, inadequate spacing of children and rearing more than one infant at a time. Although their questionnaire survey for mothers was hospital-based, their results suggested that the proportion of child maltreatment in families with twins was higher than in families with singletons. The noteworthy finding was that the twins themselves were not necessarily abused, but rather the siblings of twins. This means that having twin children can result in a reduction of the time and energy that the mother has for meaningful relationships with the father and other siblings within the family unit [1].

On the other hand, Nelson and Martin [3] reported that of 310 registered abused/neglected children, 16 (5.2\%) were twins, which was about 2.5-fold higher than the approximated general percentage of twins (2\%). They concluded that twins themselves were also at high risk, supporting the findings of Nakou et al. [7], which showed that 4 out of 50 registered abused children were twins. It is not surprising that multiples themselves are at high risk, since multiples had many general risk factors for child maltreatment, for example, low-birthweight, prematurity, birth defects, neonatal complications and so on. According to the nationwide hospital-based data provided in 1986 by Tanimura et al. [4], of 231 children subjected to abuse or neglect, $23(10.0 \%)$ were products of multiple births (22 were twins). They compared this percentage to that of twin deliveries (number of mothers) in the general Japanese population $(0.6 \%)$. They should have compared the percentage with that of live multiple births, since their research interest was the risk of being abused as a twin, not the risk of abuse occurring in families with twins. According to the vital statistics, the percentage of multiple live births among total live births in 1986 was $1.4 \%$. The percentage of twins in the maltreated population was, thus, around 7-fold $(=10.0 / 1.4)$ higher than in the general population.

It is important to note that the ratio of the percentage of specific factors in fatal child maltreatment cases to the percentage in the general population, for example, the percentage of multiple births in fatal child maltreatment cases divided by the percentage of multiple births in the general population at birth, does not yield the correct estimation of RR. This method gives an alternative underestimation of RR, since this method did not consider the percentage of the singletons (unexposed) population and the age of the subjects, although the degree of underestimation seemed not to be fatal. This method has been used several times in studies of the child maltreatment of twins [3, 4].

Using the data presented by Luke and Brown [8], the percentages of total maltreatment deaths before 1 year of age among singletons and multiple births from 1995 to 2000 in the US were recalculated as $0.0232 \%(=4,325 /$ $18,636,575)$ and $0.0607 \% \quad(=47 / 77,460)$, respectively, which produced an RR of 2.62 with $95 \%$ CI 1.96-3.49 per child. This value is slightly higher than the present result, but not higher than that estimated by Tanimura et al. [4], although the age distribution of the victims was very different. The difference between the present data, the data of Luke and Brown [8] and the data of Tanimura et al. [4] was that the former two data sets corresponded to fatal child maltreatment, i.e., child deaths, and the latter corresponded to survivors of maltreatment admitted to the hospital. The higher proportion of twins in the data of Tanimura et al. [4], however, was not rationally explained by this difference in the data. One possible explanation is that multiples in general might be admitted into the hospital compared to singletons due to other reasons than child maltreatment, thus they were apt to be over-ascertained. More research should be performed on multiple-birth status among the survivors of child maltreatment.

Most previous clinical studies focused on multiple births per child. This is not necessarily appropriate from the public health or preventive medical point of view, because most difficulties in child rearing related to multiple births were due to the rearing of more than one child of the same age at the same time in the same family [5, 9-11]. For example, the comparison of two infants (twins) consisting of one low-birthweight twin and one non-low-birthweight 
twin sometimes is a source of stress for mothers. These anxieties or feelings of stress may not be induced if rearing only one low-birthweight singleton. If multiple births were treated as individual births, the associated risk of rearing two or more children of the same age at the same time in the same family would be underestimated. The rapid increase of iatrogenic multiple births is now a public health concern, one that goes beyond purely obstetric problems [12]. Nevertheless, this serious situation is rarely recognized not only among child support members, but also among professionals in the field of parent and child health and even in families with multiples themselves [12].

According to the vital statistics, the total fertility rate tended to decrease and fell to below two over a long period of time in Japan. This suggests that the risk of having at least one maltreated baby in one family may become higher in families with multiples, which have at least two children, than in families with singletons.

The present results also showed that teenage pregnancy was a significant risk factor for fatal child maltreatment. Luke and Brown [8], using US vital statistics, showed an increased risk of infant maltreatment deaths among healthy, full-term infants among those born to mothers aged 24 and younger.

Most of the limitations of the present study could be attributed to the data collection system itself. Although this study was based on the annual reports of nationwide survey, the data gathering was far from comprehensive. The very high percentage of missing values of all three risk factors showed the difficulties of gathering data on child maltreatment. The present RR should be interpreted as the general tendency of these three risk factors.

Many of the problems that occur during pregnancy and the perinatal periods are associated with one another. For example, multiple births are associated with many perinatal problems, such as low-birthweight, Caesarean section, neonatal asphyxia, impending abortion/threatened premature delivery and pregnancy hypertension. For example, about $70 \%$ of multiples are low-birthweight in Japan [5]. Being a member of a multiple could be considered an additional risk factor for low-birthweight. The present aggregation data cannot permit multivariate analyses restricting the confounding factors.

According to the recent report by Schnitzer et al. [13], no single data source was adequate to provide thorough surveillance of fatal child maltreatment, but combining just two sources substantially increased case ascertainment. Unfortunately, most record linkage, including that between birth records and child maltreatment, was almost impossible in Japan. The assumption that the percentage of the exposed population in the general population was constant for children from birth to 17 years of age, which was made in the calculation of RR was not necessarily appropriate.
The percentage of the exposed group might gradually decrease with age, since the children in the exposed group would die more frequently compared to the children in the unexposed group because for reasons other than child maltreatment, especially at an earlier age. This seemed, however, to have little effect of the present results, since fatal child maltreatment is very rare, and the mortality rate of children themselves is extremely low in Japan.

In conclusion, recent Japanese nationwide data showed that families with multiple births had elevated risk for fatal child maltreatment, but this risk was not as high as previously thought. Multiple births should be considered a risk factor for child maltreatment, not only per individual child, but also per family unit. Health care providers should be aware that multiple pregnancies/births may place significant stress on a family, and they should provide appropriate support and intervention beginning with pregnancy as a potential high-risk group.

Acknowledgments I would like to thank Toshimi Ooma and Chieko Temba for assistance with data analysis and useful suggestions. This work was supported in part by a Grant-in-Aid for Scientific Research (B) (Grant Number 24390167) from the Japan Society for the Promotion of Science.

Conflict of interest The author declares no conflict of interests.

\section{References}

1. Robarge JP, Reynolds ZB, Groothuis JR. Increased child abuse in families with twins. Res Nurs Health. 1982;5:199-203.

2. Groothuis JR, Altemeier WA, Robarge JP, O'Connor S, Sandler $\mathrm{H}$, Vietze $\mathrm{P}$, et al. Increased child abuse in families with twins. Pediatrics. 1982;70:769-73.

3. Nelson HB, Martin CA. Increased child abuse in twins. Child Abuse Negl. 1985;9:501-5.

4. Tanimura M, Matsui I, Kobayashi N. Child abuse of one of a pair of twins in Japan. Lancet. 1990;336:1298-9.

5. Ooki S. The effect of an increase in the rate of multiple births on low-birth-weight and preterm deliveries during 1975-2008. J Epidemiol. 2010;20:480-8.

6. UNICEF. A league table of child maltreatment deaths in rich nations. (Innocenti Report Card 5). Florence: UNICEF Innocenti Research Centre; 2003.

7. Nakou S, Adam H, Stathacopoulou N, Agathonos H. Health status of abused and neglected children and their siblings. Child Abuse Negl. 1982;6:279-84.

8. Luke B, Brown MB. Maternal risk factors for potential maltreatment deaths among healthy singleton and twin infants. Twin Res Hum Genet. 2007;10:778-85.

9. Bryan E. The impact of multiple preterm births on the family. BJOG. 2003;110:24-8.

10. Ellison MA, Hotamisligil S, Lee H, Rich-Edwards JW, Pang SC, Hall JE. Psychosocial risks associated with multiple births resulting from assisted reproduction. Fertil Steril. 2005;83: 1422-8.

11. Pinborg A, Loft A, Schmidt L, Andersen AN. Morbidity in a Danish national cohort of 472 IVF/ICSI twins, 1132 non-IVF/ 
ICSI twins and 634 IVF/ICSI singletons: health-related and social implications for the children and their families. Hum Reprod. 2003;18:1234-43.

12. Ooki S. Effect of maternal age and fertility treatment on the increase in multiple births in Japan: vital statistics, 1974-2009. J Epidemiol. 2011;21:507-11.
13. Schnitzer PG, Covington TM, Wirtz SJ, Verhoek-Oftedahl W, Palusci VJ. Public health surveillance of fatal child maltreatment: analysis of 3 state programs. Am J Public Health. 2008;98: 296-303. 